\title{
Undergraduate course on biomedical imaging at a liberal arts college
}

\section{Michael Durst}

Michael E. Durst, "Undergraduate course on biomedical imaging at a liberal arts college," Proc. SPIE 11143, Fifteenth Conference on Education and Training in Optics and Photonics: ETOP 2019, 111430R (2 July 2019); doi: $10.1117 / 12.2522197$

SPIE Event: Fifteenth Conference on Education and Training in Optics and Photonics: ETOP 2019, 2019, Quebec City, Quebec, Canada 


\title{
Undergraduate Course on Biomedical Imaging at a Liberal Arts College
}

\author{
Michael E. Durst ${ }^{\mathrm{a}}$ \\ ${ }^{a}$ Middlebury College, Middlebury, VT 05753, USA
}

\begin{abstract}
This paper presents an intermediate-level undergraduate course on the physical principles of biomedical optics and imaging. Through in-class labs, Mathematica simulations, field trips, and group presentations, students learn about fundamental imaging concepts in optical microscopes. After developing an understanding of the role of the Fourier transform in image formation, the course shifts to non-optical imaging, including x-ray computed tomography, ultrasound, and magnetic resonance imaging. The significance of this course is its handson nature, and this paper offers examples of laboratory exercises and simulations to promote active learning in the classroom.
\end{abstract}

Keywords: biomedical optics, imaging, undergraduate course, lab exercises, Mathematica simulations

\section{INTRODUCTION}

In this paper, I present an intermediate-level physics course on biomedical imaging, with the goal of sharing resources to aid in the development of similar undergraduate optics courses. ${ }^{1-6}$ I introduced this course for three reasons: to provide an interdisciplinary physics course to support a liberal arts education, to attract students who are underrepresented in physics to the major, and to bring my research on biomedical optics into the classroom. Beyond the students' interest in the subject matter, this course works well because the physical phenomena are both visual and hands-on in nature, although simulations and a field trip to a hospital radiology department are required for most non-optical imaging techniques.

Beginning with the study of geometric optics, students explore the concepts of image formation by building a microscope from scratch. This topic facilitates learning about reflection, refraction, interference, diffraction, resolution, aberration, and the point spread function. After understanding these fundamental imaging concepts, students are ready to explore biomedical optics, including standard optical microscopes, fluorescence imaging, spectroscopy, fiber-optic endoscopes, and laser-scanning microscopes. Because visible light does not penetrate very deeply into the human body, we then shift wavelengths to study x-ray radiography and computed tomography $(\mathrm{CT})$. Finally, we investigate imaging techniques that do not involve light, such as ultrasound, nuclear medicine, and magnetic resonance imaging (MRI). Table 1 shows a list of topics covered during the 12-week semester.

The course emphasizes the role of the Fourier transform in all these biomedical imaging modalities. With a pre-requisite requirement of multivariable calculus, students dive into the calculations of resolution and image formation. The Fourier transform first appears in our derivation of Fraunhofer diffraction, which leads to a discussion of how lenses perform Fourier transforms. This helps explain how Köhler illumination works and where conjugate planes can be found in a microscope. Students also use the Fourier transform to understand resolution in both the spatial domain (the point spread function) and the spatial frequency domain (the optical transfer function). After using optics to understand the Fourier transform, students grasp the significance of the Fourier transform in non-optical imaging, namely the projection-slice theorem in computed tomography and the use of frequency encoding to acquire an MRI image in frequency space.

For topics not covered in the course, students give group presentations. I scaffold this project by spreading it out over the course of the semester, having the students first choose topics, then write outlines, and submit a

Further author information:

M.E.D.: E-mail: mdurst@middlebury.edu

Fifteenth Conference on Education and Training in Optics and Photonics: ETOP 2019, edited by

Anne-Sophie Poulin-Girard, Joseph A. Shaw, Proc. of SPIE Vol. 11143, 111430R · (c) 2019

SPIE, ICO, IEEE, OSA·CCC code: 0277-786X/19/\$18 · doi: 10.1117/12.2522197

Proc. of SPIE Vol. 11143 111430R-1 
Table 1. Topics covered in the course, with each row in the detail column corresponding to one 75-minute class meeting.

\begin{tabular}{|c|c|c|}
\hline part & topic & detail \\
\hline \multirow{16}{*}{ 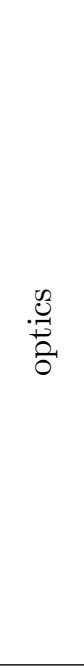 } & \multirow{3}{*}{ waves } & wave characteristics \\
\hline & & vectors, electromagnetic waves \\
\hline & & plane waves, spherical waves, Gaussian beams \\
\hline & \multirow{3}{*}{ geometric optics } & reflection, refraction, Fresnel equations \\
\hline & & lenses, image formation \\
\hline & & ray tracing matrices, aberrations \\
\hline & \multirow{4}{*}{ physical optics } & wave interference \\
\hline & & diffraction, Huygens-Fresnel principle \\
\hline & & Fraunhofer diffraction \\
\hline & & Fresnel diffraction, resolution \\
\hline & \multirow{3}{*}{ Fourier optics } & Fourier optics, Fourier transform \\
\hline & & point spread function, convolution \\
\hline & & optical transfer function \\
\hline & \multirow{2}{*}{ sources of contrast } & blackbody radiation, lasers, energy levels \\
\hline & & fluorescence, absorption, scattering \\
\hline & modern optical microscopy & confocal, two-photon, super-resolution \\
\hline \multirow{5}{*}{ 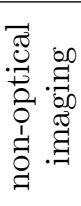 } & x-ray & $\mathrm{x}$-ray generation, $\mathrm{x}$-ray radiography \\
\hline & computed tomography (CT) & projections, Radon transform \\
\hline & magnetic resonance & nuclear magnetic resonance (NMR) \\
\hline & imaging (MRI) & pulse sequences, k-space acquisition \\
\hline & ultrasound & reflection, refraction, A-mode \\
\hline \multirow{3}{*}{$\frac{\vec{d}}{\overrightarrow{0}}$} & field trip & radiology department at local hospital \\
\hline & \multirow{2}{*}{ projects } & student presentations day 1 \\
\hline & & student presentations day 2 \\
\hline
\end{tabular}

draft presentation. In addition, I meet with the students for a practice presentation before their final draft, giving them feedback on their presentation content and style. Students rarely receive training in oral presentations, and they greatly appreciate the opportunity to improve their oral presentations. The students also really enjoy exploring topics of their interest, which include functional MRI, photoacoustic imaging, total internal reflection fluorescence microscopy, optogenetics, x-ray microscopes, and positron emission tomography.

In addition to traditional lectures, this course employs several pedagogical best practices, including active learning techniques and field trips. Using a clicker response system, the students answer concept questions during class using their smartphones or laptops, allowing students to participate when they might not otherwise feel comfortable speaking up in front of their peers. By challenging the students to actively participate during lecture time, I am able to more deeply engage the students and create a classroom atmosphere focused on understanding and retention. The class also takes a 2.5-hour tour of the radiology department at Porter Medical Center in Middlebury, VT. The students meet with technicians and radiologists in charge of CT, MRI, ultrasound, and single-photon emission computed tomography, using real data to explain how the machines work. Lastly, we take three field trips within our science building, McCardell Bicentennial Hall: a tour of my lab to observe a custom-built laser-scanning two-photon microscope, a tour of the fluorescence microscopes in the cell imaging facility, and a tour of the nuclear magnetic resonance (NMR) spectrometer facility.

One of the biggest challenges of teaching this course to an undergraduate audience is finding an appropriate textbook which covers all the desired topics at an appropriate level of mathematics. For instance, many biomedical imaging textbooks either skip the math or use general forms without concrete examples or calculations. After assigning readings from multiple textbooks in a first iteration of the course, I now use my class notes typeset as a textbook, using LaTeX and an open source textbook class file developed by Peatross and Ware. ${ }^{7}$ Many textbooks proved to be useful resources for different aspects of the course. For optics, resources include Optics by Hecht, ${ }^{8}$ Introduction to Optics by Pedrotti et al.,${ }^{9}$ Principles of Physical Optics by Bennett, ${ }^{10}$ Fundamen- 
tals of Photonics by Saleh and Teich, ${ }^{11}$ Laser Electronics by Verdeyen, ${ }^{12}$ and Physics of Light and Optics by Peatross and Ware, ${ }^{7}$ as well as introductory physics textbooks like Essential University Physics by Wolfson. ${ }^{13}$ For biomedical optics in particular, Fundamentals of Biomedical Optics by Boudoux ${ }^{14}$ and From Photon to Neuron: Light, Imaging, Vision by Nelson ${ }^{15}$ both offer excellent perspectives. For non-optical biomedical imaging, the resource which best matches our level of mathematics is Medical Imaging: Signals and Systems by Prince and Links. ${ }^{16}$ Additional resources include Introduction to Physics in Modern Medicine by Kane, ${ }^{17}$ The Essential Physics of Medical Imaging by Bushberg et al. ${ }^{18}$ Introduction to Medical Imaging by Smith and Webb, ${ }^{19}$ and Fundamental Mathematics and Physics of Medical Imaging by Lancaster and Hasegawa. ${ }^{20}$

In the following sections, we outline the laboratory exercises and Mathematica ${ }^{21}$ simulations used in the course, and we provide detailed examples of each.

\section{LABORATORY EXERCISES}

The course meets twice per week for 75 minutes each, allowing for the lecture period to alternate between inclass labs, clicker questions, and traditional lecture. A list of lab exercises is shown in Table 2. These hands-on activities are essential to understanding the more advanced material in the course. Much of the exercises center around optics, using optical breadboards, rails, lenses, HeNe lasers (Thorlabs), and CCD cameras (Lumenera). Typically, the students assemble each activity themselves, allowing them to master laser beam alignment using kinematic mirror mounts and alignment irises. For the microscope activities, we borrow a fleet of older Olympus BH2 microscopes retired by the biology department. Also, we study laser-scanning techniques like confocal microscopy and two-photon microscopy using galvo mirrors (Industrial Fiber Optics) to scan the laser beam. For non-optical imaging, we have found excellent ideas in the literature. To demonstrate computed tomography, we use a simple Pasco photogate following the experiment from Mylott et al. ${ }^{22}$ The photogate, which consists of a NIR LED on one side and a detector on the other, is slid across a sample on a rotation stage, creating binary (as opposed to gray scale) CT image projections recorded in LabView. In addition, we use an ultrasound kit from TeachSpin which allows us to record A-scans with a transducer, ${ }^{4,23,24}$ and we share the output with multiple oscilloscopes for the students to analyze. For other non-optical techniques for which it would be too costly to have labs, we use Mathematica-based computer simulations and animations, as well as a field trip to a local hospital.

In the following subsections, we give detailed descriptions of two lab exercises: the build-your-own microscope activity and the resolution and modulation transfer function activity.

\subsection{Lab Exercise - Build-Your-Own Microscope}

In this lab, students investigate the image formation properties of lenses by assembling two microscope configurations: a compound microscope for observation with the human eye (Fig. 1), and an infinite-conjugate microscope for CCD camera detection (Fig. 2). The students locate any intermediate and final image planes and identify each image as real or virtual, upright or inverted, and enlarged or reduced. In addition, the students qualitatively observe aberrations in their image, allowing them to appreciate the design of commercial objective lenses for high quality images. The quantitative component of the lab involves drawing ray tracing diagrams and calculating the magnification of the system.

The most challenging aspect of the experiment is the calculation of angular magnification for the virtual image formed by the compound microscope. While students intuitively understand that an object appears larger when moved closer to the observer, quantifying the angular magnification creates much difficulty. The students can quickly plug values into the magnification equation for a compound microscope and get a theoretical value: $m=-(L \cdot 25 \mathrm{~cm}) /\left(f_{o b j} \cdot f_{\text {eyepiece }}\right)$, where the $25 \mathrm{~cm}$ comes from the near point of the eye, $L$ is the distance between the two lenses, and $f_{o b j}$ and $f_{\text {eyepiece }}$ are the focal lengths of the objective lens and eyepiece, respectively. However, to measure the angular magnification experimentally, the students must obtain the ratio of the angle $\beta$ subtended by the object when viewed through the lenses compared to the angle $\alpha$ when it is held at the near point and viewed without a lens (Fig. 1 (b)). This ratio of angles $m=\beta / \alpha$ can be inferred by measuring the height of the object and its image if both are located the same distance from the observer. The sticking point is asking the students to use calipers to measure the size of a virtual image which they cannot touch; they must alternately peer over and through the lenses while adjusting the calipers in mid-air to match the size of 
Table 2. List of in-class labs, simulations, and demonstrations, along with a brief description.

\begin{tabular}{|c|c|c|}
\hline part & lab & description \\
\hline \multirow{16}{*}{ 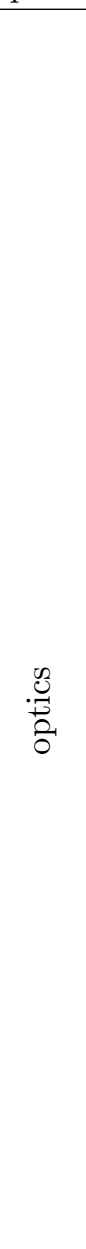 } & intro to Fourier transforms & $\begin{array}{l}\text { detect sound frequencies using a microphone } \\
\text { and an oscilloscope in FFT mode }\end{array}$ \\
\hline & Gaussian beams & $\begin{array}{l}\text { photograph and analyze a laser beam spot } \\
\text { with ImageJ }\end{array}$ \\
\hline & Snell's law & $\begin{array}{l}\text { measure angles of incidence, refraction, } \\
\text { and reflection with a protractor }\end{array}$ \\
\hline & Fresnel equations & $\begin{array}{l}\text { measure the power of incident, refracted, } \\
\text { and reflected beams }\end{array}$ \\
\hline & imaging with a lens & $\begin{array}{l}\text { observe images formed by a single lens } \\
\text { on an optical rail }\end{array}$ \\
\hline & build-your-own microscope & $\begin{array}{l}\text { combine lenses to create a microscope } \\
\text { and observe its aberrations }\end{array}$ \\
\hline & double-slit interference & $\begin{array}{l}\text { observe a double-slit interference pattern } \\
\text { formed by various slit separations }\end{array}$ \\
\hline & single-slit diffraction & $\begin{array}{l}\text { observe single-slit diffraction } \\
\text { with various slit widths }\end{array}$ \\
\hline & Airy disk & $\begin{array}{l}\text { photograph diffraction from a circular aperture } \\
\text { and analyze it with ImageJ }\end{array}$ \\
\hline & resolution & $\begin{array}{l}\text { observe the diffraction limit in a microscope } \\
\text { using Ronchi rulings }\end{array}$ \\
\hline & Fourier optics & $\begin{array}{l}\text { spatial filtering in the Fourier plane } \\
\text { with variable apertures }\end{array}$ \\
\hline & point spread function & $\begin{array}{l}\text { image microspheres with a microscope } \\
\text { and a camera }\end{array}$ \\
\hline & optical transfer function & $\begin{array}{l}\text { observe the modulation of different spatial } \\
\text { frequencies in a microscope using Ronchi rulings }\end{array}$ \\
\hline & laser-scanning microscopy & $\begin{array}{l}\text { build a reflection confocal microscope } \\
\text { using a galvo scanning mirror }\end{array}$ \\
\hline & fluorescence & $\begin{array}{l}\text { use a USB spectrometer to measure the } \\
\text { fluorescence spectra of dyes }\end{array}$ \\
\hline & advanced optical microscopy & $\begin{array}{l}\text { observe samples in fluorescence, phase contrast, } \\
\text { and differential interference contrast (DIC) mode }\end{array}$ \\
\hline \multirow{5}{*}{ 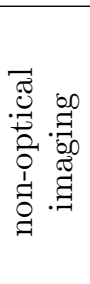 } & $\mathrm{x}$-ray radiography & projection simulation \\
\hline & computed tomography (CT) & $\begin{array}{l}\text { computed tomography with a photogate, } \\
\text { rotation stage, and LabVIEW }{ }^{22}\end{array}$ \\
\hline & nuclear magnetic resonance (NMR) & field trip, magnetization vector simulation \\
\hline & magnetic resonance imaging (MRI) & Bloch equation simulation \\
\hline & ultrasound & $\begin{array}{l}\text { ultrasound A-mode scans with a transducer } \\
\text { and an oscilloscope } \mathrm{e}^{4,23}\end{array}$ \\
\hline
\end{tabular}

the virtual image. Also, if the image is not located at the near point, then students assume that they made a mistake when their experimental and theoretical values do not match.

For camera detection with the infinite-conjugate microscope, students really appreciate being able to see a real image both on a card and on the computer screen via the camera. First, the distance between the camera and the tube lens is set by imaging a distant object, i.e., an object at infinity. Then, the objective lens is inserted with a separation of the sum of the focal lengths of the objective and tube lens. Lastly, the object is mounted on a slider and shifted until in focus on the camera. For the lateral magnification, the students experimentally measure the distances both with calipers and by counting pixels in ImageJ, and they compare their values to the theoretical result $M=-f_{t u b e} / f_{o b j}$.

The required equipment includes lenses of various focal lengths, an optical rail, Vernier calipers, a flashlight, 

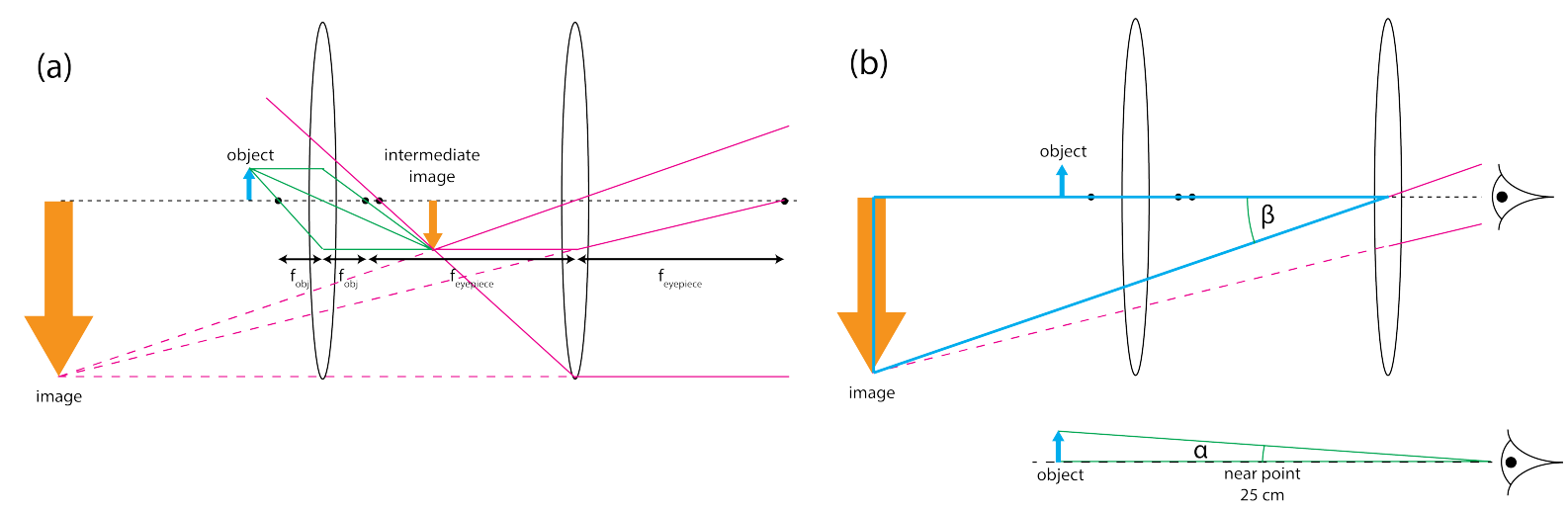

Figure 1. Build-your-own microscope lab: compound microscope exercise. (a) Ray diagram of the compound microscope. (b) Diagram of angle $\beta$ subtended by image as viewed through the microscope compared to the angle $\alpha$ subtended when the object is held at the near point.

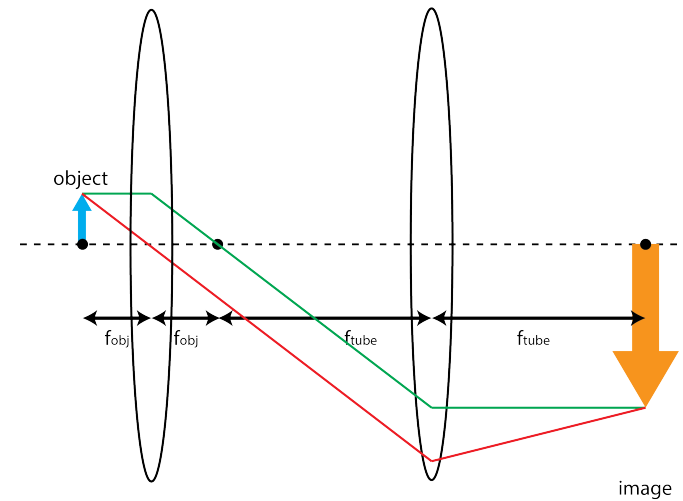

Figure 2. Build-your-own microscope lab: infinite-conjugate microscope ray-tracing diagram.

and a CCD camera attached to a computer. For an object with fine features, we use either an Air Force target or a business card in which the inkjet dots can be observed. To reduce the cost of the experiment, the CCD camera sensor can be replaced by either a smartphone or laptop camera, so long as the built-in lens is taken into account as part of the optical layout.

\subsection{Lab Exercise - Resolution and the Modulation Transfer Function}

In this lab, students determine the resolution of a commercial microscope using Ronchi rulings viewed through various objective lenses. In addition, students measure the modulation transfer function and determine the cutoff spatial frequency, allowing students to think about resolution in the Fourier domain. Students compare their results to the Rayleigh criterion for resolution $0.61 \cdot \lambda / N A$ and the cutoff frequency expression $2 \cdot N A / \lambda$, where $N A$ is the numerical aperture of the objective lens.

In the first exercise, students determine the resolution of an Olympus BH2 microscope qualitatively using their eyes. The turret is equipped with $4 \mathrm{x} / 0.10,10 \mathrm{x} / 0.25,20 \mathrm{x} / 0.40$, and $40 \mathrm{x} / 0.65$ objectives, which correspond to the expected resolutions for $\lambda=500 \mathrm{~nm}$ listed in Table 3. For an object with various fine features of known size, we use a set of fine Ronchi rulings (Edmund Optics, $400 \mathrm{lp} / \mathrm{mm}$ and $600 \mathrm{lp} / \mathrm{mm}$ ) as well as a variable line grating slide (Thorlabs, from $1.25 \mathrm{lp} / \mathrm{mm}$ to $250 \mathrm{lp} / \mathrm{mm}$ ). As shown in Table 3, for our set of Ronchi rulings, we are able to observe the resolution limit only for the $4 \mathrm{x}$ and $10 \mathrm{x}$ objectives.

In the second exercise, students calculate the amplitude of the optical transfer function, known as the modulation transfer function (MTF), by observing the blurring which occurs at higher spatial frequencies. To measure the MTF, the student must measure how well each spatial frequency is passed through the system. To do 
Table 3. Expected resolution and cutoff frequencies for objectives with various numerical apertures at a wavelength of 0.5 $\mu \mathrm{m}$. The Ronchi ruling frequency is converted from the cutoff frequency using a factor of 2 to account for the line pairs.

\begin{tabular}{|c|c|c|c|}
\hline objective & resolution $(\mu \mathrm{m})$ & $\begin{array}{c}\text { cutoff frequency } \\
(1 / \mu \mathrm{m})\end{array}$ & $\begin{array}{c}\text { Ronchi ruling } \\
(\mathrm{lp} / \mathrm{mm})\end{array}$ \\
\hline $4 \mathrm{x} / 0.10$ & 3.1 & 0.4 & 200 \\
\hline $10 \mathrm{x} / 0.25$ & 1.2 & 1.0 & 500 \\
\hline $20 \mathrm{x} / 0.40$ & 0.76 & 1.3 & 800 \\
\hline $40 \mathrm{x} / 0.65$ & 0.47 & 2.6 & 1300 \\
\hline
\end{tabular}

this, we use the variable line grating and Ronchi rulings as the sample and measure the modulation for each frequency; each value corresponds to one data point on the MTF curve. The amount of modulation for one spatial frequency is defined to be ${ }^{8}\left(I_{\max }-I_{\min }\right) /\left(I_{\max }+I_{\min }\right)$, where $I_{\max }$ is the maximum intensity in the modulation and $I_{\min }$ is the minimum. Experimentally, we attach a CCD camera to the microscope using an eyepiece adapter (Fig. 3(a)). By drawing line profiles across the image in ImageJ (Fig. 3(b)), we can quickly calculate the modulation value for each Ronchi ruling.

(a)

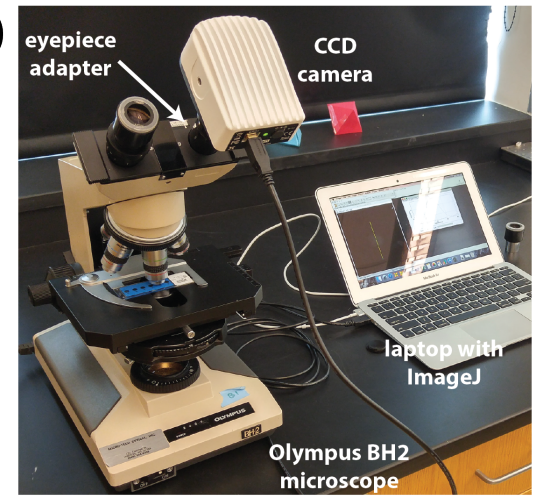

(b)

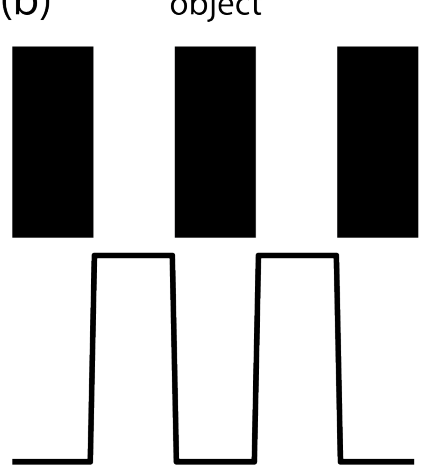

image
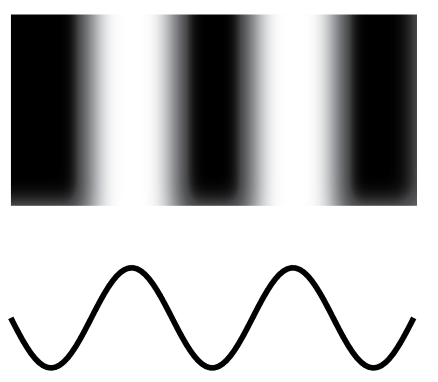

Figure 3. Resolution and modulation transfer function lab. (a) Photograph of microscope with CCD camera attached via an eyepiece adapter. (b) Schematic of a line grating (top) and its corresponding line profile (bottom) before and after passing through the system.

The biggest challenge for students is maintaining the sharpest possible image. For physics majors, this is their first experience observing a thin, fine sample with a commercial microscope, requiring fine adjustments to the sample stage focus as well as optimizing the eyepiece for relaxed viewing. In addition, they are attempting to measure intensity fluctuations that are highly sensitive to alignment as well as the uniformity of the illumination. This lab is typically preceded by an exercise on Köhler illumination, and the students are expected to repeat an illumination checklist at the start of lab.

These lab activities use an optical microscope with several objectives, a set of Ronchi rulings, an eyepiece to C-mount adapter, and a CCD camera attached to a computer with ImageJ. To reduce the cost of this activity, a custom-built microscope made of singlet lenses would have a resolution limit which can be obtained using Ronchi rulings generated with a laser printer, and the cutoff frequency can be determined by eye or with a laptop or smartphone camera.

\section{MATHEMATICA SIMULATIONS}

Visualizations are essential to student understanding of optics and biomedical imaging, so the course incorporates several Mathematica simulations written both by the instructor as well as by the student for homework. Animations of plane waves, Gaussian beams, and spherical waves reveal how light propagates in free space and at a boundary. Students create their own ray tracing program using ray matrices. Interference and diffraction 
patterns from slits, rectangular apertures, and circular apertures are quickly plotted to better understand the derived expressions. Fourier transforms of the apertures reveal the same diffraction patterns, and fun calculations like convolution and correlation can be easily performed in Mathematica. For non-optical imaging, Mathematica simulations are crucial. We show the results of a fan x-ray projection, the Radon transform in CT, saturation recovery and spin echo in MRI, and beam steering and focusing in ultrasound.

Here, we provide two examples of Mathematica simulations used in the course: spatial filtering in the Fourier domain and parallel-ray projections and backprojections in CT.

\subsection{Simulation - Spatial Filtering in the Fourier Domain}

In this Mathematica example (Appendix A), we perform spatial filtering of the Middlebury 'M' in the Fourier domain. Using the Manipulate function, students can apply several masks: a low-pass filter of varying size (white circle), a high-pass filter of varying size (black circle), block half the vertical frequencies (horizontal black and white bars), block half the horizontal frequencies (vertical black and white bars), low pass the vertical frequencies (horizontal white strip), low pass the horizontal frequencies (vertical white strip), high pass the vertical frequencies (horizontal black strip), and high pass the horizontal frequencies (vertical black strip). A slider allows the students to change the width of each mask. In the display, the students see the original image, its Fourier transform, the mask applied in the Fourier domain, and the resulting image in the spatial domain after the mask is applied in the Fourier domain.

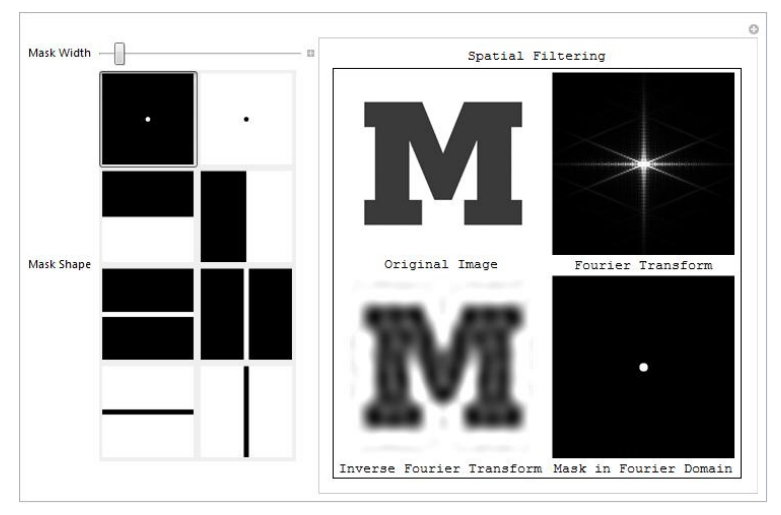

Figure 4. Screenshot from Mathematica simulation of spatial filtering in the Fourier domain. After selecting a mask (left), the program displays the original image, its Fourier transform, the mask, and the resulting image after convolving the object with the mask.

Students really enjoy applying these filters to their own images. Also, they are surprised to learn about the applications of filtering in the Fourier domain to image jpeg compression, noise filtering, and audio compression, filtering, and auto-tuning. This simulation supplements our Fourier optics laboratory exercise in which various apertures are placed at the Fourier plane of a lens system. For the complete code, see Appendix A.

\subsection{Simulation - Computed Tomography: Projections and Backprojections}

In computed tomography, parallel x-rays pass through an object, and the shadow image of these parallel rays is detected on the other side. The source and detector then rotate, acquire another shadow image, and repeat until an image has been acquired from each angle. In this Mathematica simulation (Fig. 5, Appendix B), we show the profile for one x-ray line passing through an arbitrary object (a circle object is depicted). The object function, which represents the values of the attenuation coefficients, is shown in the left panel of Fig. 5. To display all the rays parallel to this one, we can change the lateral position parameter $\rho$ with the slider. The projection is the integral of our object function along each line characterized by $\rho$, i.e., the area under each line profile curve. The projection for this particular angle $\theta$ is plotted in the box on the right in Fig. 5. For other angles, a sinogram allows a fast visualization, with each projection along each row in a $\theta$ v. $\rho$ plot. 


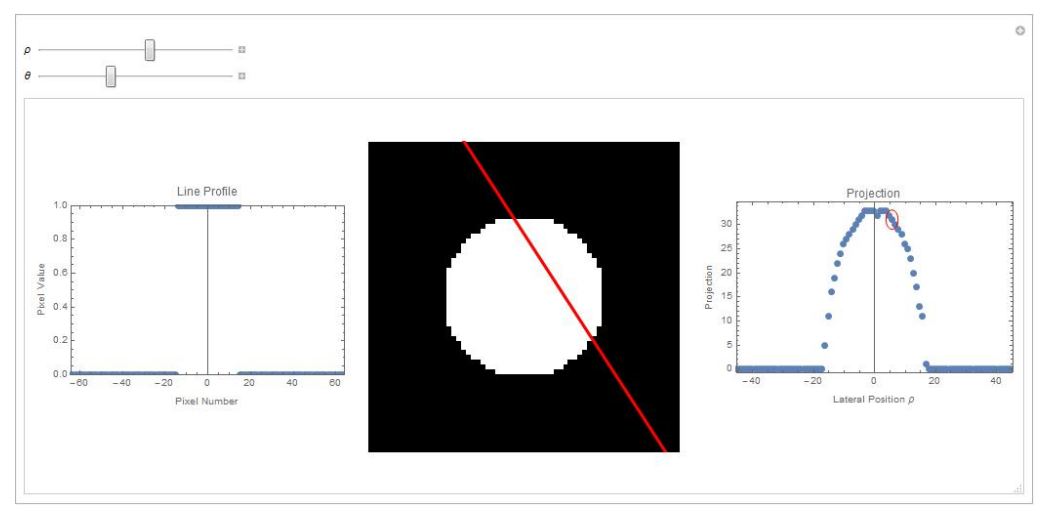

Figure 5. Screenshot from Mathematica simulation of CT projections. (left) Line profile for a given $\rho$ and $\theta$. (center) Object with current ray depicted in red. (right) Projection for a given $\theta$ found by the area under each line profile curve, with the current $\rho$ circled in red.

To reconstruct the object, a simple unfiltered backprojection gives a quick insight into the process. For a given angle $\theta$, the easiest guess for the shape of the object is to assign every point along the line at that angle the value of the projection. When overlayed with the backprojection from other angles, we can build up the backprojection summation image (Fig. 6). We then compare our discrete backprojection result with one obtained with Mathematica's built-in Radon transform function. The student can select other object shapes, which are available in the Mathematica file in Appendix B.

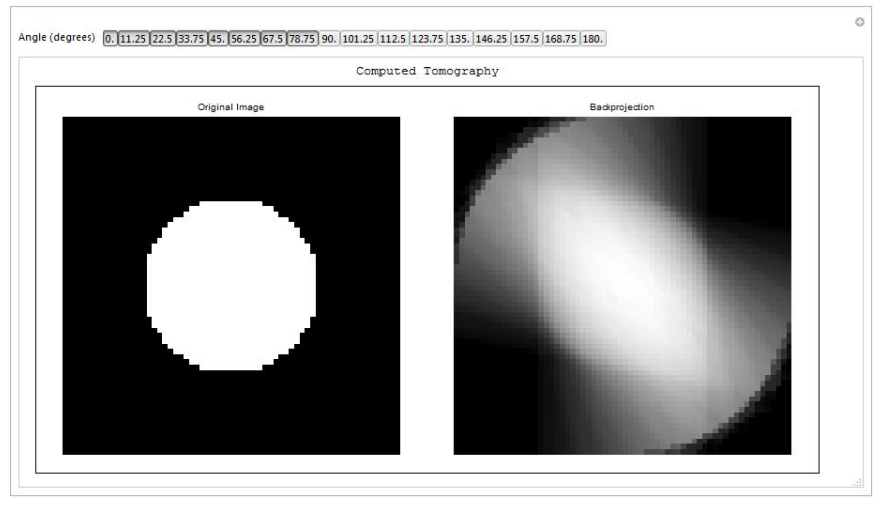

Figure 6. Screenshot from Mathematica simulation of CT backprojections. (left) Original object. (right) Backprojection summation image formed by the angles selected in the button array along the top.

\section{CONCLUSION}

I have presented an intermediate-level physics course on biomedical imaging, with an emphasis on hands-on lab activities and Mathematica simulations. I have taught the course twice in the past four years at Middlebury College, and the course has been well received by students. Students report getting the most out of working on their final presentation projects and applying their imaging knowledge to understanding new techniques which were not covered in class. Future iterations of the course will expand the Mathematica simulation offerings to include real-world medical imaging data as well as more applications of radioactivity to imaging and therapy. Because it can be difficult to get such a course off the ground, the goal of this paper is to share resources to facilitate the creation of similar undergraduate optics and biomedical imaging courses in the future. 


\section{APPENDIX A. MATHEMATICA CODE - SPATIAL FILTERING IN THE FOURIER DOMAIN}

The Mathematica code for the simulation from Sec. 3.1 is detailed here.

\section{Initiate Parallel Processing}

Needs ["CUDALink" "]

SetDirectory@NotebookDirectory []

\section{Import Image and Generate Masks}

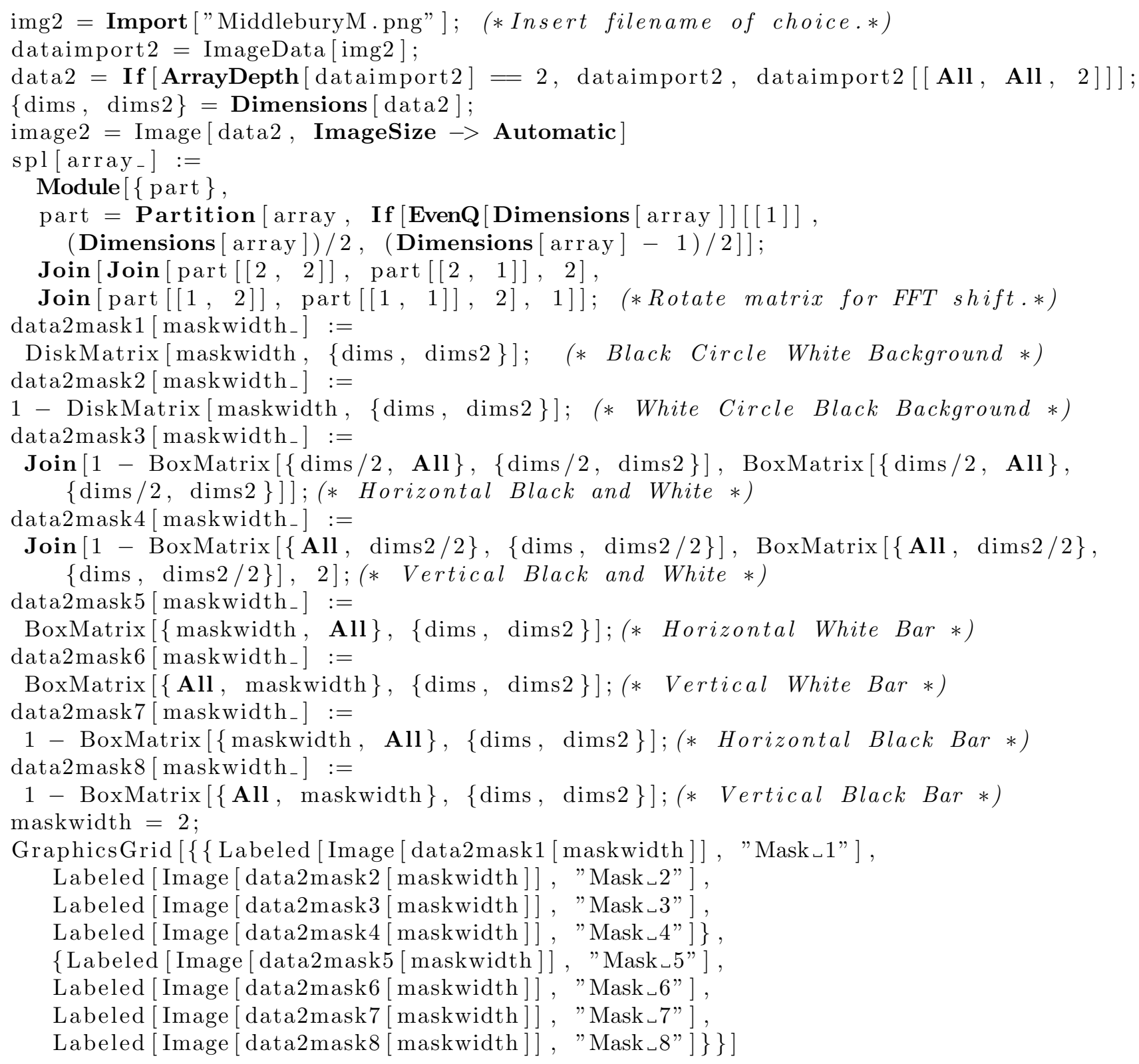

\section{Apply Mask to Image in Fourier Domain}

Select mask by clicking on shape. Adjust the width of the shape with the slider. 


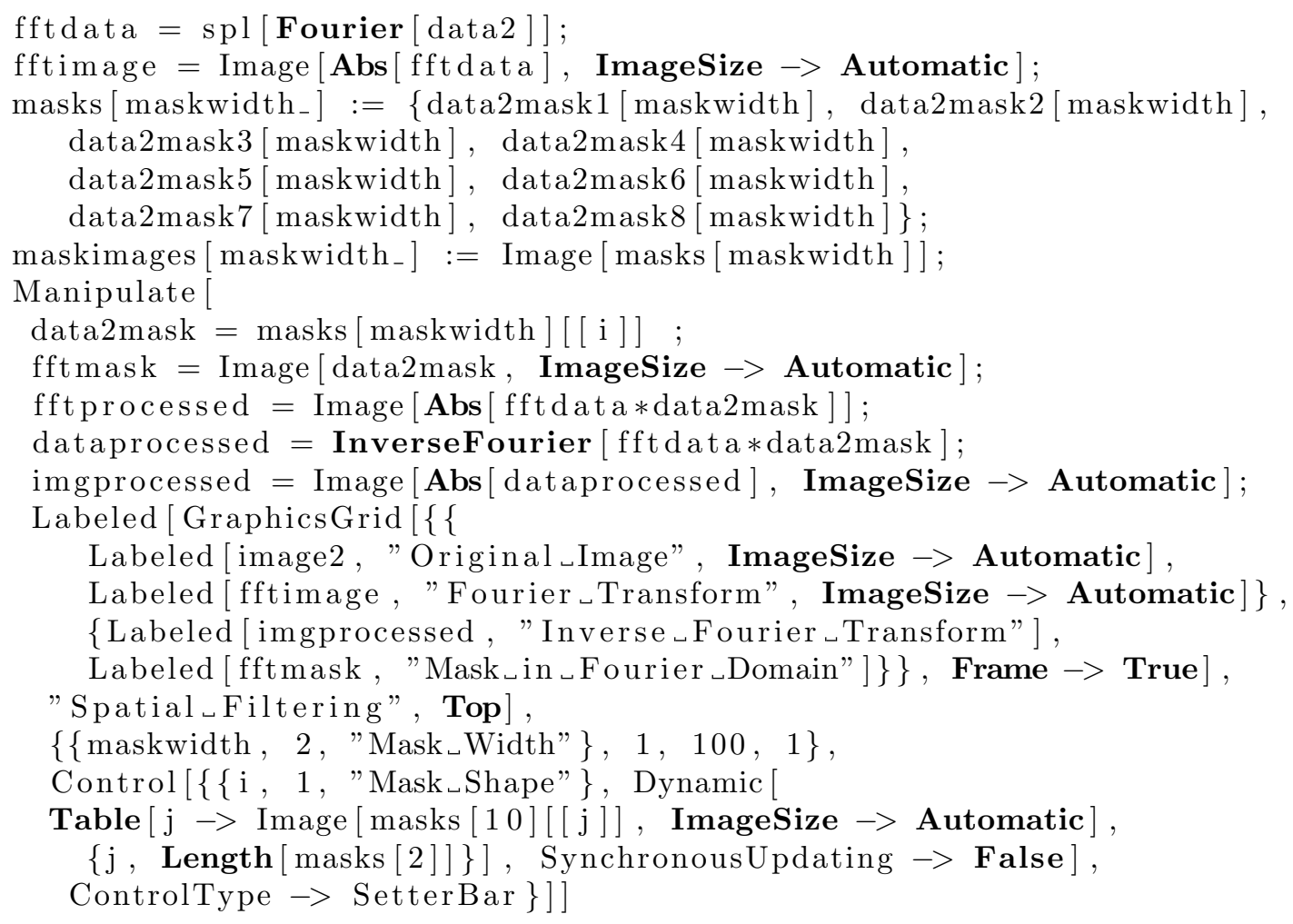

\section{APPENDIX B. MATHEMATICA CODE - COMPUTED TOMOGRAPHY: PROJECTIONS AND BACKPROJECTIONS}

The Mathematica code for the simulation from Sec. 3.2 is detailed here.

\section{Initiate Parallel Processing}

Needs ["CUDALink" "]

SetDirectory@NotebookDirectory []

\section{Choose Object}

$\operatorname{dims}=64$

objectlist $=$

List [Image [DiskMatrix [16, \{dims, $\operatorname{dims}\}]]$,

Image [DiamondMatrix [16, \{dims, dims $\}]]$,

Image [BoxMatrix $[16,\{$ dims, dims $\}]]$,

Image $[$ SparseArray $[\{\operatorname{Band}[\{1,1\}] \rightarrow 1, \operatorname{Band}[\{1,2\}] \rightarrow 1$,

Band $[\{2,1\}] \rightarrow 1\},\{\operatorname{dims}, \operatorname{dims}\}]]$,

Image [CrossMatrix $[\{16,16\},\{$ dims, $\operatorname{dims}\}]]]$

g1 = Image [objectlist [[1]]]

(*hange between 1: Disk, 2: Diamond, 3: Square, 4: Diagonal, 5:Cross*)

\section{Generate Line Profiles and Projections}

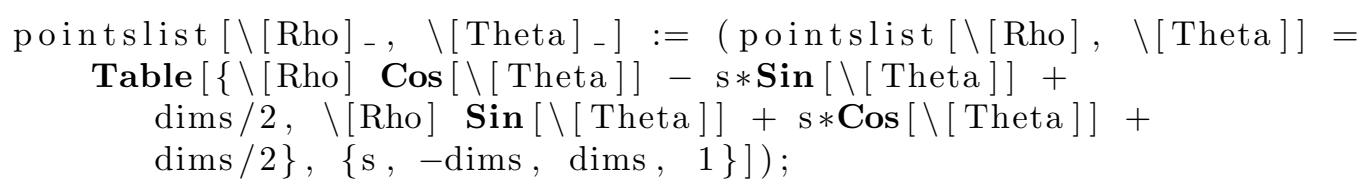




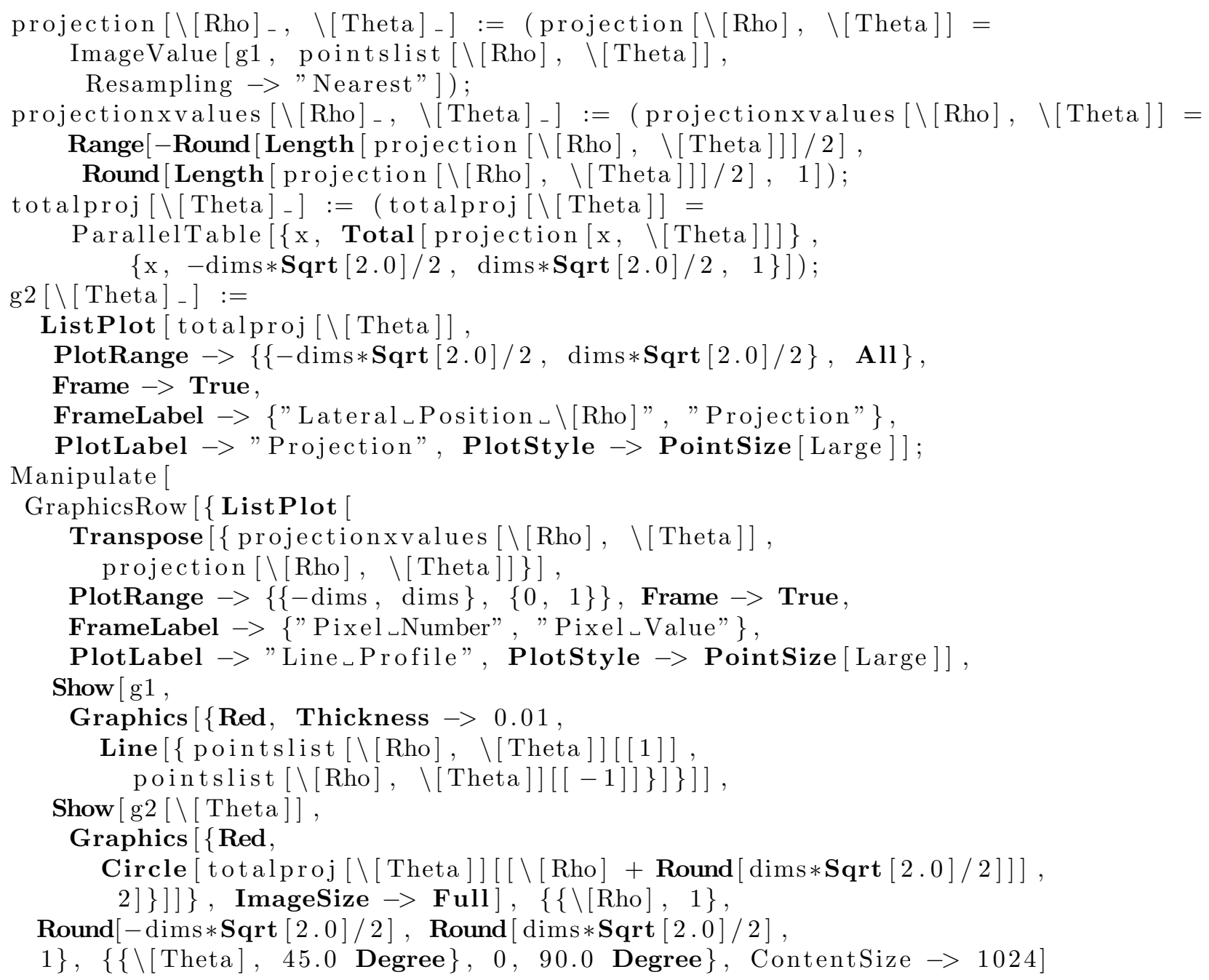

\section{Assemble Projections into a Sinogram}

sinogramdata $=$

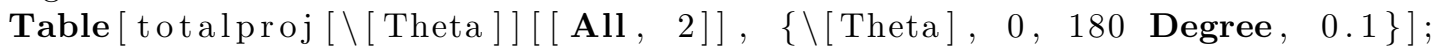
sinogramxvalues $=$ Table $[\backslash[$ Theta $],\{\backslash[$ Theta $], 0,180$ Degree, 0.1$\}]$; Manipulate [

GraphicsRow [ $\{$ Show [

ArrayPlot [sinogramdata, ColorFunction $\rightarrow$ GrayLevel,

PlotLabel $\rightarrow$ "Sinogram $(\backslash[$ Theta $]\lrcorner$ v. $\backslash \backslash$ Rho $])$ ",

AxesLabel $\rightarrow\{" \backslash[$ Rho $] ", " \backslash[$ Theta $] "\}$, ImageSize $\rightarrow$ Large $]$,

Graphics $[\{$ Red, Thickness $\rightarrow 0.01$,

Line $[\{\{0$,

Part [Position [sinogramxvalues, Round $[\backslash[$ Theta $], 0.1]], 1$,

$1]\}, \quad\{\operatorname{dims} * \operatorname{Sqrt}[2]$,

Part [Position [sinogramxvalues, Round [ \[Theta], 0.1]], 1,

ListPlot $1]\}\}]\}]]$,

sinogramdata [ [

Part [Position [sinogramxvalues, Round[ \[Theta], 0.1]], 1, 1]]],

PlotLabel $\rightarrow$ "Projection", AxesLabel $\rightarrow\{$ $>[$ Rho $]$ ", "Projection" $\}$, 


\footnotetext{
PlotStyle $\rightarrow$ PointSize $[$ Large $]]\}], \quad\{\{\backslash[$ Theta $], 0$ Degree $\}, 0$, 180 Degree, 0.1$\}]$
}

\section{Generate Backprojections}

Click on angles to add backprojection to backprojection summation image.

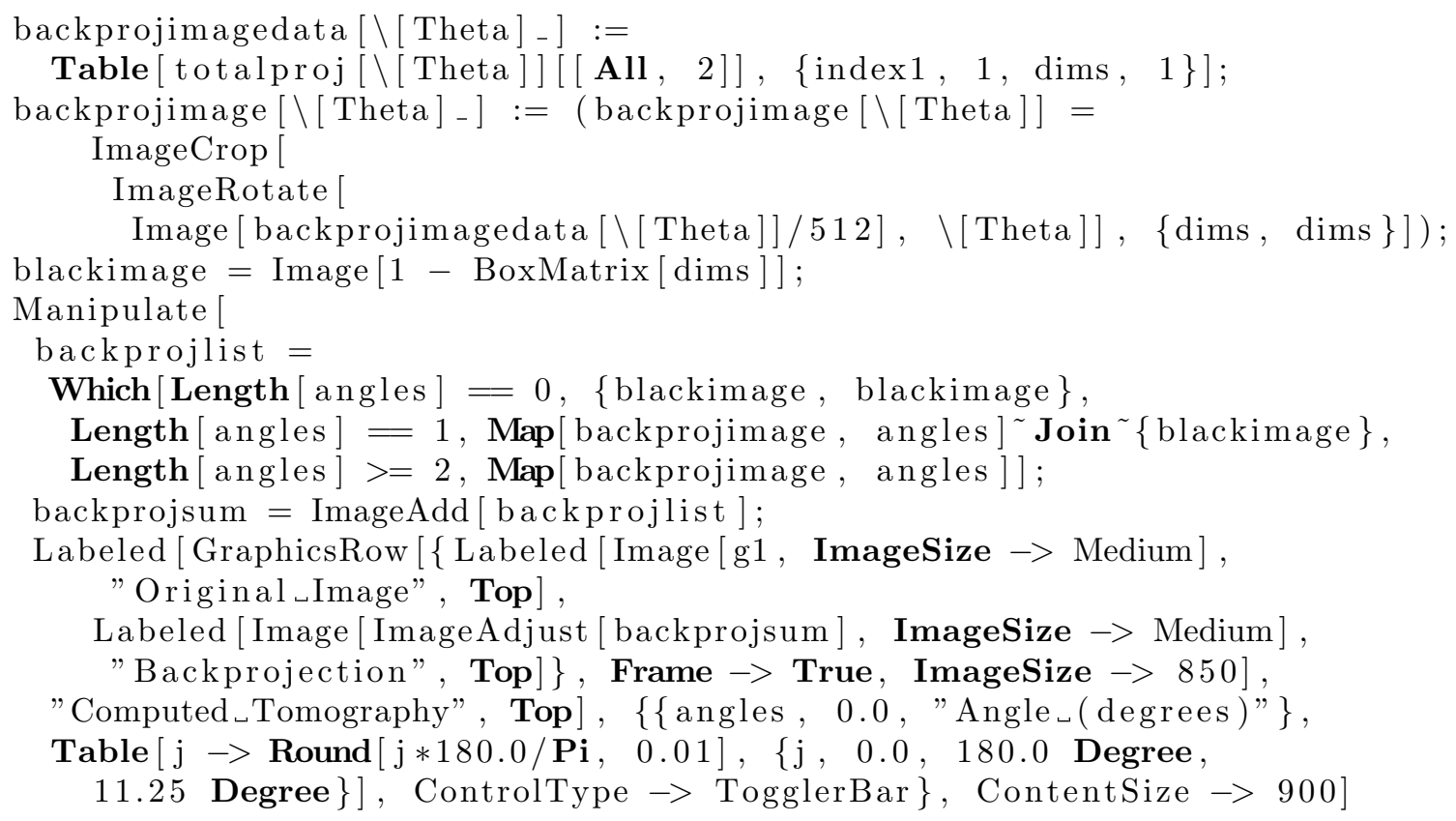

\section{Backprojections Using Built-In Radon Transform}

Adjust number of iterations with slider.

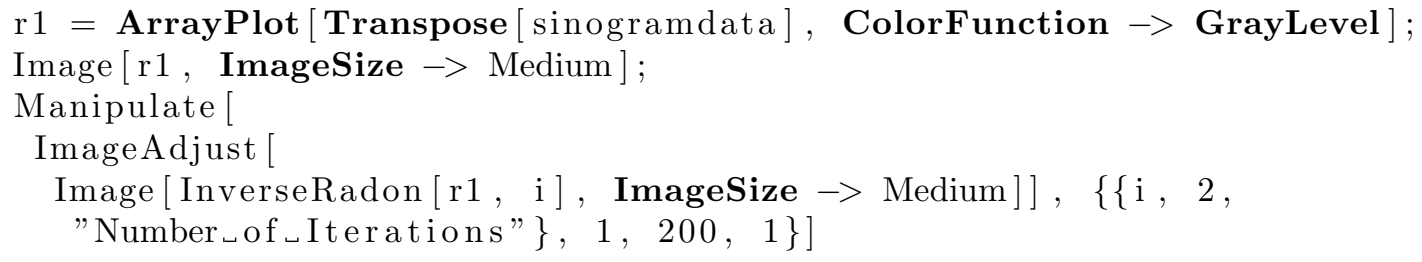

\section{ACKNOWLEDGMENTS}

Many thanks to Jeff Dunham for help in streamlining the Mathematica code and to Lance Ritchie for help in assembling the laboratory exercises. Also, thanks to Michelle Aines and the radiology department at Porter Medical Center for providing tours, demonstrations, and in-depth tutorials of the latest medical imaging technologies.

\section{REFERENCES}

[1] Scalettar, B. A. and Abney, J. R., "Biomedical imaging in the undergraduate physics curriculum: Module on optical microscopy," American Journal of Physics 83(8), 711-718 (2015).

[2] Van Ness, G. R. and Widenhorn, R., "Engaging the community through an undergraduate biomedical physics course," American Journal of Physics 80(12), 1094-1098 (2012).

[3] Christensen, N., "Medical physics: the perfect intermediate level physics class," European Journal of Physics 22(4), 421 (2001).

[4] Amador Kane, S., "An undergraduate biophysics program: Curricular examples and lessons from a liberal arts context," American Journal of Physics 70(6), 581-586 (2002). 
[5] Paschal, C. B., Nightingale, K. R., and Ropella, K. M., "Undergraduate biomedical imaging education," Annals of Biomedical Engineering 34(2), 232-238 (2006).

[6] Zollman, D., Jones, D., Murphy, S., v.d. Wirjawan, J., and Norvell, N., "Teaching about the physics of medical imaging: Examples of research-based teaching materials," Lat. Am. J. Phys. Educ. Vol 1(122), 6 (2012).

[7] Peatross, J. and Ware, M., [Physics of Light and Optics], self published (2015).

[8] Hecht, E., [Optics], Pearson Education, Incorporated (2017).

[9] Pedrotti, F., Pedrotti, L., and Pedrotti, L., [Introduction to Optics], Cambridge University Press (2017).

[10] Bennett, C., [Principles of Physical Optics], Wiley (2008).

[11] Saleh, B. and Teich, M., [Fundamentals of Photonics], Wiley Series in Pure and Applied Optics, Wiley (2007).

[12] Verdeyen, J. T., [Laser Electronics], Prentice Hall series in solid state physical electronics, Prentice Hall (1995).

[13] Wolfson, R., [Essential University Physics], vol. 2, Pearson Education (2020).

[14] Boudoux, C., [Fundamentals of Biomedical Optics], Blurb, Incorporated (2017).

[15] Nelson, P., [From Photon to Neuron: Light, Imaging, Vision], Princeton University Press (2017).

[16] Prince, J. and Links, J., [Medical Imaging Signals and Systems], Pearson (2015).

[17] Kane, S., [Introduction to Physics in Modern Medicine], CRC Press (2009).

[18] Bushberg, J., Seibert, J., Leidholdt, E., and Boone, J., [The Essential Physics of Medical Imaging], Wolters Kluwer Health (2011).

[19] Smith, N. and Webb, A., [Introduction to Medical Imaging: Physics, Engineering and Clinical Applications], Cambridge Texts in Biomedical Engineering, Cambridge University Press (2010).

[20] Lancaster, J. and Hasegawa, B., [Fundamental Mathematics and Physics of Medical Imaging], Series in Medical Physics and Biomedical Engineering, CRC Press (2016).

[21] Wolfram, "Mathematica, Version 12.0," (2019). Champaign, IL, 2019.

[22] Mylott, E., Klepetka, R., Dunlap, J. C., and Widenhorn, R., "An easily assembled laboratory exercise in computed tomography," European Journal of Physics 32(5), 1227 (2011).

[23] TeachSpin, I., "Ultrasonics, advanced physics lab experiments," (accessed April 29, 2019). https://www. teachspin.com/ultrasonics.

[24] Stiles, T. A., "Ultrasound imaging as an undergraduate physics laboratory exercise," American Journal of Physics 82(5), 490-501 (2014). 